\title{
Overview: Human-Computer Interaction an Globally Used Technique in Society
}

\author{
Nidhi Manchanda, Sanatan Jha \\ B.Tech, Computer Science \\ \& Engineering \\ Dronacharya College \\ of Engineering, Gurgaon, India \\ Email: \{nidhi.17092, sanatan.17127\} \\ (a) ggnindia.dronacharya.info
}

\author{
Sarita \\ Assistant Professor, \\ Computer Science \& Engineering \\ Dronacharya College of \\ Engineering \\ Gurgaon, India \\ Email: sarita10103@gmail.com
}

\author{
Dr. Saurabh Mukherjee \\ Computer Science, \\ Associate Prof. \\ Banasthali University \\ Rajasthan, India \\ Email: mukherjee.saurabh \\ (a)rediffmail.com
}

\begin{abstract}
IThis paper discussed about the basic terms related to human computer interaction which will help us to gather a detail knowledge and review on terms, existing technologies and latest coming technology of the subject which include different configurations. Use of computer always has a question that how to interact with it, if used properly then can help humans to work very efficiently and faster. A brief outline of HCI is displayed. Particular cases of research in the ranges of symbols and menus are then surveyed.

Study of topics in it covered HCI framework, Existing technologies, Issues, application and trends in HCI.
\end{abstract}

Index Terms-Indian sign Language (ISL), Human Computer Interaction (HCI), Graphical user Interface (GUI)

\section{InTRODUCTION}

HCI (Human-Computer Interaction) is a technique which defines the ways that how humans interact with computers and how humans can use them efficiently. In simple words, interaction between human and computer defines the term HCI. The term HCI (Human-Computer Interaction) was introduced in one of the book of 1983, The Psychology of Human-Computer Interaction which is based upon a research by Stwart K.Card and Allen Newell of Carnegie University. The term HCI (Human-Computer Interface) is a study that provides the different methods of evaluation, interaction and design for computing systems. HCI is itself a very wide scope and include disciplines like psychology, sociology, anthropology, cognitive science, computer science, and linguistic. In todays, modern world invention of speech interaction with computers is also a part of HCI.

$\mathrm{HCI}$ is the term with many alternative terms and also be called as Human-Machine Interaction (HMI), which means the interaction of humans with computers, codes and algorithms are designed by humans which are followed by computers to complete the task in the particular manner they need to follow. Secondly it is referred as Man-Machine Interaction (MMI), which also means the communication of man or human with computer machine to execute different programs formed by man. There is no difference of the meaning if it is referred as Computer-Human Interaction
(CHI) too, which indicates the interaction of computer which is a machine with human. The names are given to the term according to its functionality.

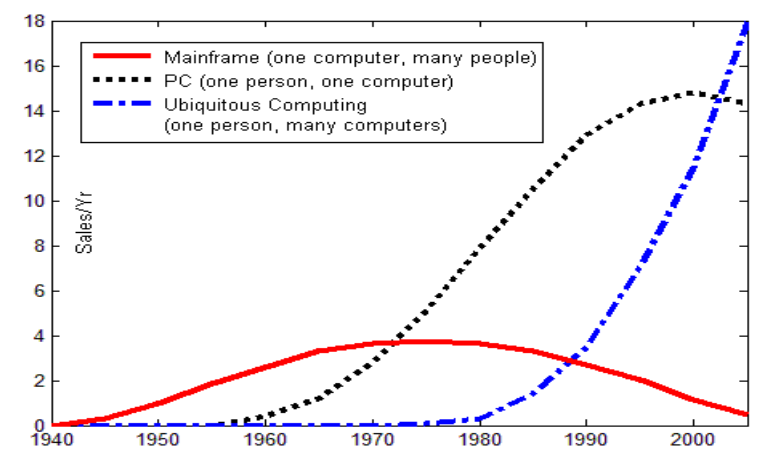

Fig 1: Major trends in computing

This Figure deals with the growth of computer and human interaction. The graph shows us how the computers have increased the role in the human life and influence human life. Now days each one of us use computer to complete there day to day work more easily and efficiently. There is an increase in the graph showing the increased number of computer per Human Being.

\section{Goals OF HCI}

In the beginning, there were humans. 'Computers' came in 1940s and the term HCI came in 1980s. What about the time interval 1940-1980. In those days computers were too complicated and to simply that compilation is the man goal of HCI.

\section{A. HCI (HUMAN-Computer Interaction): Terminologies}

uman-Computer Interaction is a strong area of research and results into emerging of new technologies, which increase scope of interaction of human with machine. It defines the communication method with which user and computer interact with each other. Loop of interaction can 
be known on the basis of flow of information between user and computer.

The loop of interaction between user and computer depends upon many aspects. The basic aspect is task environment, it affect whole interaction process of machine with user depending upon what user want to execute using any algorithm, other are Areas of the Interface on which user want to work with machine, Input flows which user give to the machine, Output which user receive from the computer after providing input, Feedback of the machine depending on the demands of the user and Fit [2].

The most basic terms involve in it are: Functionality and Usability. With the designing of any system the first thing come in mind is what will be its function? What is the use of it? Set of services and actions that are provided to the users are called as functionality of the system. Functionality can only be valued and visible when it is properly used by user. Some functionality by which a system is used is the measure of degree by which a system [3].

Term HCI was first used in 1980 and also was known in 1975.Humans or users get interact with computer majorly due to desktop applications. The limited icons space at desktop tends the user to communicate or interact with computer. The further interaction starts due to trend of internet browsers and handheld computers.

\section{B. The interfaces between humans and computers: \\ 1. GUI (Graphical User Interface) \\ 2. VUI (Voice User Interface)}

GUI deals with all graphical interfaces between users and computers, icons, desktops, images all these are dealt through it.

If user wants any kind of speech recognition and systems synthesizing between him and computer then he can use VUI (Voice User Interface) technology [4].

WIMP (Windows, icons, Menus and Pointing devices) interface (Desktop Metaphor of GUI) is also a standard interface between machine (computer) and man.

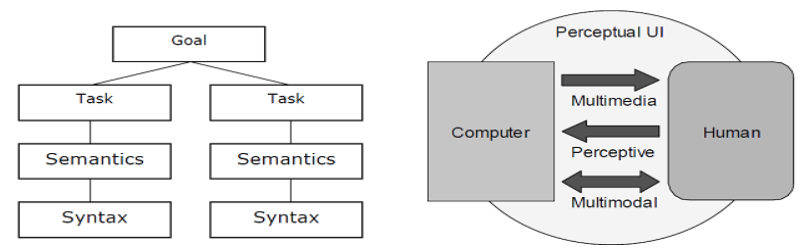

Fig 2: Flowchart of Overview in $\mathrm{HCI}$
This Figure deals with the overview of Human Computer interaction which helps to know how to communicate and share videos, data, and information and exchange various multimedia with the computer. This provides a basic view of Human Computer Interaction.

\section{Overview on HCI}

In 1970's, information technology professionals and dedicated hobbyists were the only humans who communicate with computer machine. In later 1970's, emergence of personal computing changed the scenario disruptively and include personal computer and personal software resulting increase in potential of computer user and also highlighted the limitations of computer compared to its usability for the users who want them as tools[5].

Computer plays a crucial role in everybody's life and had changed the way people live over the last two decades. HCI technology is becoming so important and changing the lives of humans that in near future no task or ambition can be fulfilled without computing technology.

The module removes highlight from hand picture for signal acknowledgment and extricated elements will be food as info for acknowledgment process. The state of the form is a vital property that can be utilized to recognize hand motions. Forms of hand picture are extricated as highlight. These shapes are put away as form layouts which will be utilized for motion acknowledgment.

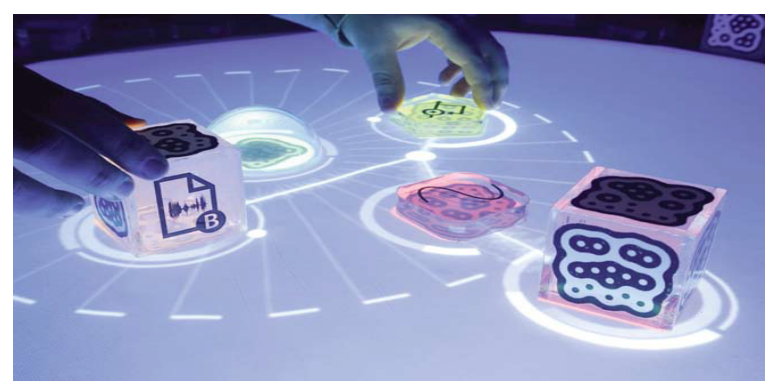

Fig 3: Extraction (Indian sign Language) of Information from Gestures.

The extraction suggests highlight vector of isolated picture which can be isolated in different strategies for application. Investigator portrayal of highlight extraction and a couple of methodology rely on upon frame and range based shape representation. 


\section{EXISTING TECHNOLOGIES OF HCI:}

The main aspect to be considered is human behaviour and needs to be useful. The focus of this paper is mostly on the advances in physical aspects of interaction can be obtained (Multi -Modal Interaction) and how each method can be improved in performance (Intelligent Interaction) to provide a better and easy interface for the user. The existing physical technologies of $\mathrm{HCI}$ basically can be categorized by the relative human sense that the device is designed for. These devices are basically relying on three human senses: vision, audition, and touch [7].

The input devices that rely are either switch-based or pointing devices. The output devices can be any kind of visual display or printing device. Architecture of HCI showcases these inputs and outputs it generates. Some developing configurations and designs on which interfaces today are based:

1. Unimodal Interaction Systems

1.1. Audio Based HCI

1.2. Visual Based HCI

1.3. Sensor Based HCI

2. Multimodal Interaction Systems [8].

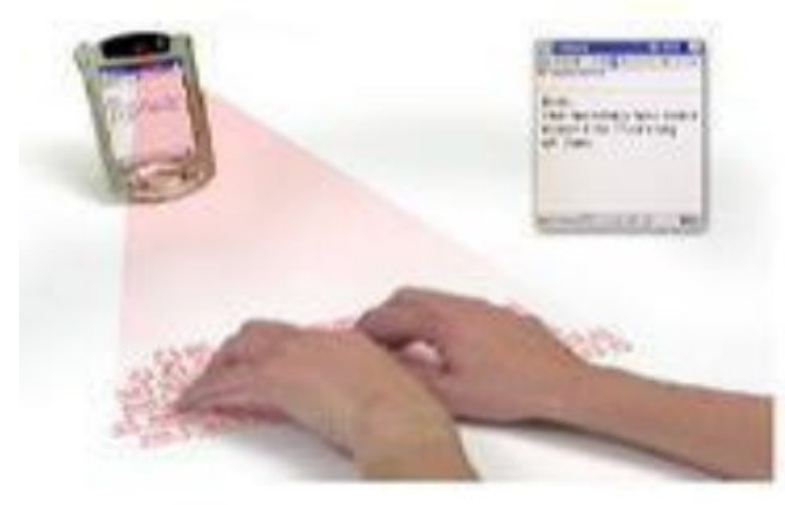

Fig 4: Canesta virtual keyboard

\section{A glimpse on HCI Issues :}

This topic is not considered much but Eason (1991) proposed a three level model for it. Includes perceptual principles,
I. Legible displays
II. Top-down processing

III. Avoidance of absolute judgment limits

IV. Redundancy gain

\section{Similarity causes confusion}

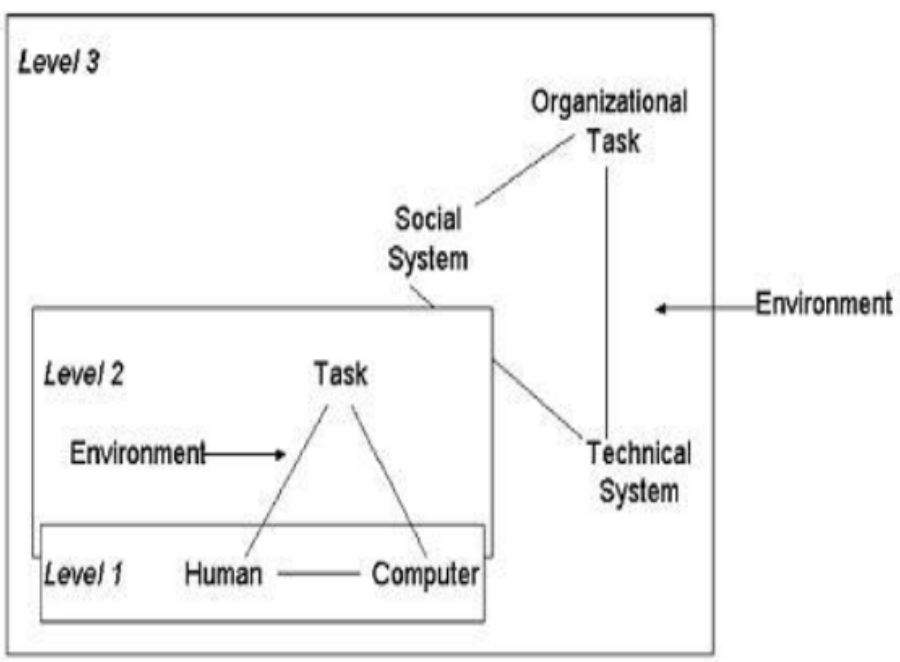

Fig 5: A Three level model of HCI

Level 1 consists of two participants' information processing i.e. direst interaction between humancomputer. Level 2 shows their interaction with environment to perform different tasks. Level 3 shows effects of these interactions on world.

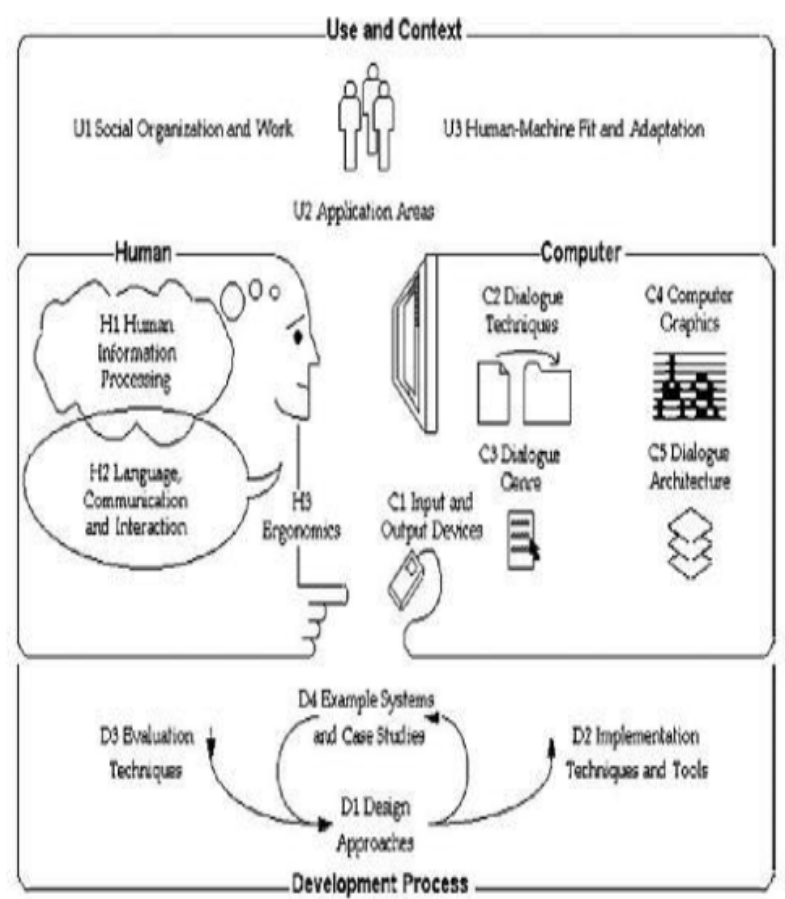

Fig 6: ACM SIGCHI curricula for HCI 


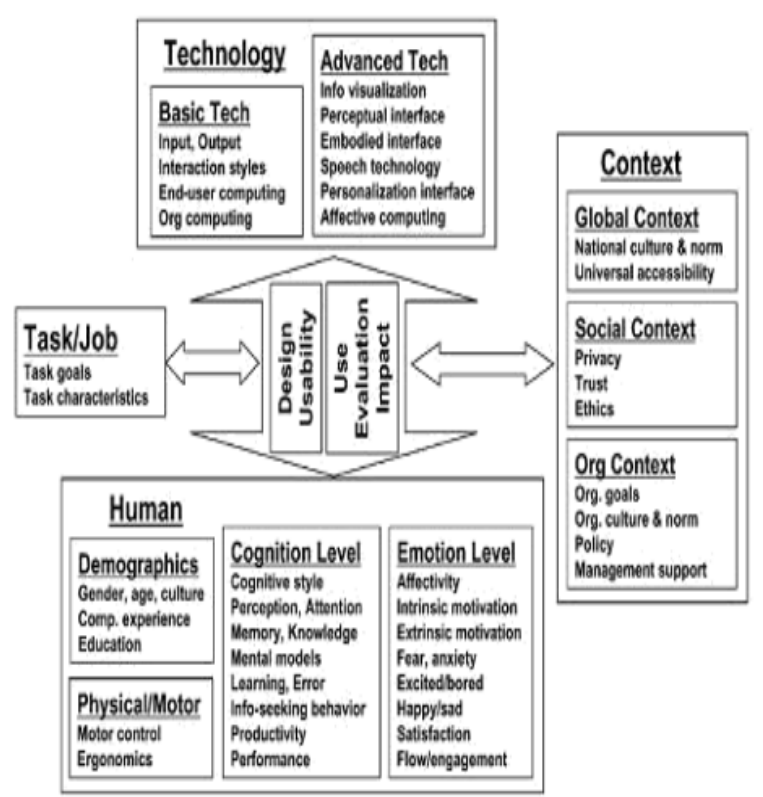

Fig 7: A framework of broad HCI issues and concerns.

\section{PEOPLE AND MULTIMEDIA}
I. Perceptive User Interfaces
II. Multimodal User Interfaces
III. Multimedia User Interfaces

In order to endow computers with similar capabilities, we need significant progress in several technologies, including:

- Speech and sound recognition

- Natural language understanding

- Computer vision

- Dialog management/planning

- Learning

- User modeling

- Haptic

The motions which are required must be characterized ahead of time. For instance on the off chance that we are utilizing a communication via gestures, the vocabulary should be characterized. Portray every single motion as far as HMM. The structures of the move capacity yield likelihood thickness capacity are assessed in this progression. Preprocessing the information includes transient Fourier change and vector quantization. Here the information is gathered and signal is characterized through preparing information. Hence this information should be spoken to in a brief structure. At that point the estimation procedure is utilized utilizing some calculation for ex. Bacum-Welch calculation. This calculation is utilized to locate the obscure parameters of Hidden Markov Model. After this, the acknowledgment of signal is done utilizing calculation Viterbi calculation (say). This calculation is utilized to locate the no doubt succession of the HMM. It is all the more entirely helpful for utilizing this calculation for the yield.

\section{LATEST TECHNOLOGIES OF HCI:}

The advancement in technologies of HCI involves recent directions and advances of research in $\mathrm{HCI}$, namely intelligent and adaptive interfaces and ubiquitous computing are presented. These interfaces involve different levels of user activity: physical, cognitive, and affection [9]. Menu designing is the major topic of research under HCI.

\section{Sensor-Based Technologies}

It has wide range of applications with at least one physical sensor between user and machine.

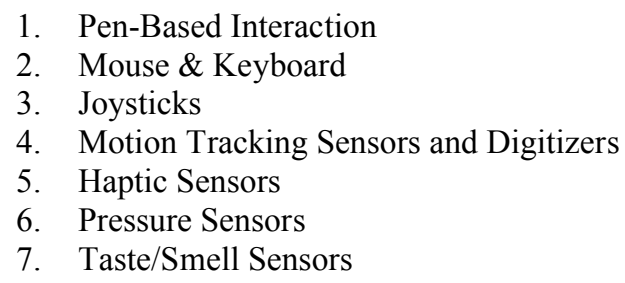

\section{AUdIO-BASED HCI}

This part of acquiring information deals with different audio signals.

Speech Recognition

Speaker Recognition

Auditory Emotion Analysis

Human-Made Noise/Sign Detections (Gasp, Sigh, Laugh, Cry, etc.)

Musical Interaction

\section{Visual-Based HCI}

This area deals with facial expressions.

1. Facial Expression Analysis

2. Body Movement Tracking (Large-scale)

3. Gesture Recognition

4. Gaze Detection (Eyes Movement Tracking) 


\section{HCI RESEARCH:}

Research under HCI concerns that, how people will simplify their task using GUI versus a text-based commandline interface? Second thing is Latency, its presence occur with internet connection. How human will do their task with internet in easier way. It needs to be more concerned about societies and human values.

\section{Conclusion:}

Human-Computer Interaction is the term which is required where computer system is used; they fulfill need of system design, interface of system with user. HCI gives birth to virtual reality. In the near future, VR with HCI will form the common interface to interact with user. It will take the world to its new horizons including intelligence, innovation and interaction techniques. We tried our best to provide the complete overview through this paper.

\section{Trends In Human Computer Interaction :}

Human-PC association is a multidisciplinary investigates range concentrated on cooperation modalities amongst people and PCs; once in a while, the more broad term human-machine interface (HMI) is utilized to allude to the UI in an assembling or process-control framework. At the end of the day, the HCI teach the ways to handle the issues related to the interface and framework between user and computer. The main aim of Human-Computer Interaction intrinsically involve different controls over software engineering, the term enhance can be identified with a few angles, including instinct of utilization and interface heartiness. An instinctive, normal, proficient, powerful, and adaptable interface can incredibly diminish the hole across user intelligence modal and the working method of any PC, system, computer, or any robot. In spite of the fact that learns about HCI go back to 1975; late innovative advances in shopper hardware have opened energizing new situations: motions, hand and body postures, discourse, and look are only a couple of normal collaboration modes that can be utilized to outline moderate common UIs (NUIs).

\section{REFERENCES}

[1] J. Y. Chai, P. Hong and M. X. Zhou, "A probabilistic way to deal with reference determination in multimodal UIs", Proceedings of the ninth International Conference on Intelligent User Interfaces, Funchal, Madeira, Portugal, pp 70-77 (2012).

[2] A. Chapanis, Man Machine Engineering, Wadsworth, Belmont (2002).

[3] B. Shneiderman, "Past smart machines:just do it!" IEEE Software, vol. 10, 1, Jan 2006

[4] B. Reeves and C. Nass, The Media Equation: HowPeople Treat Computers, Television, and New Media Like Real People and Places, Cambridge University Press,September 1996.

[5] Thoren, C. (1993). Nordic rules for PC openness. The Nordic Committee on Disability, Stockholm: The Swedish Handicap Institute.

[6] Stephanidis, C., Salvendy, G., et al. (1998). Toward an Information Society for All: An International R\&D Agenda. Universal Journal of Human-Computer Interaction

[7] Institute for Personalized Information Environment (2013). FRIEND21 Human Interface Architecture Guidelines. Tokyo, Japan.

[8] Culnan, M. J. (2010). Mapping the scholarly structure of MIS, 19801985: a co-reference examination. MIS Quarterly, 11(3), 341-353.

[9] Alavi, M., and Carlson, P. (1992). A survey of MISresearch and disciplinary advancement. Diary of Management Information Systems, $8(4), 45-62$.

[10] Barki, H., Rivard, S., and Talbot, J. (1988). A data frameworks watchword grouping plan. MIS Quarterly, 12(2), 299-322.

[11] Eason, K. D. (1991). Ergonomic point of view on advances in humancomputer cooperation. Ergonomics, 34(6), 721-741.

[12] Goodhue, D. L. (1997). The model basic the estimation of the effects of the IIC on the end-clients. Diary of the American Society for Information Science, 48(5), 449-453.

[13] Goodhue, D. L. (1998). Improvement and estimation legitimacy of an errand innovation fit instrument for client assessments of data frameworks. Choice Sciences, 29(1), 105-137.

[14] Microsoft, "Being Human HCI 2020", Research Report, March 2007, Spain

[15] P. R. Cohen, D. R. McGee, "Substantial Multimodal Interfaces for Safety-basic Applications," Communications of the ACM, Vol. 47, Issue 1, pp. 41-46, January 2004. 